\title{
Lasers with distributed loss have a sublinear output power characteristic: supplementary material
}

\author{
Tobias S. Mansuripur ${ }^{1}$, Guy-Mael de Naurois ${ }^{2}$, Alexey Belyanin $^{3}$, And \\ FEDERICO CAPASSO ${ }^{2, *}$ \\ ${ }^{1}$ Department of Physics, Harvard University, Cambridge, MA 02138 USA \\ ${ }^{2}$ School of Engineering and Applied Sciences, Harvard University, Cambridge, MA 02138 USA \\ ${ }^{3}$ Department of Physics and Astronomy, Texas A \& M University, College Station, Texas 77843 USA \\ *Corresponding author: capasso@seas.harvard.edu
}

Published 15 January 2015

This document provides supplementary information to "Lasers with distributed loss have a sublinear output power characteristic," http://dx.doi.org/10.1364/optica.2.000048. In Sec. 1 we demonstrate two different limits in which the results of the nonuniform treatment approach those of the distributed loss approximation. In Sec. 2 we explain how soon after threshold the asymptotic regime is reached. In Sec. 3 we provide mathematical details on the intracavity intensity varia-tion in a laser cavity.

(C) 2015 Optical Society of America

http://dx.doi.org/10.1364/optica.2.000048.s001

\section{LIMITING CASES OF NONUNIFORM GAIN SATURATION TREATMENT}

A. $R_{1}, R_{2} \rightarrow 1,1-R \ll \alpha_{0} L$

When the reflectivity of both mirrors approaches one and there is also non-zero distributed loss (so that $1-R \ll$ $\alpha_{0} L$ ), Eqs. 8 and 9 can be Taylor expanded to first order in the parameters $1-R_{1}$ and $1-R_{2}$, which yields the total output power

$$
\beta^{\text {out }} \approx \frac{1-R}{\alpha_{0}}\left(g_{0}-\alpha_{0}\right)
$$

The DLA yields the same result as Eq. S1 when $\beta_{\text {DLA }}^{\text {out }}$ in Eq. 4 is expanded in the same limits. The agreement of the two theories in this limit is due to the highly uniform intracavity intensity (as a result of the high mirror reflectivities) together with the fact that $\ln (1 / R) \approx 1-R$, so that the distributed out-coupling of the DLA is not a bad approximation of the true lumped nature of the mirror losses.
B. $\alpha_{0}=0$

When $\alpha_{0}=0$ there is no finite limiting value of the intracavity intensity $\beta^{\max }$; still, this is not enough of a reason to expect the DLA to be a good approximation because there can be significant intensity variation in the cavityparticularly for small $R$-that would seem to necessitate the nonuniform gain saturation treatment. Taking the limit of Eqs. 8 and 9 as $\alpha_{0}$ goes to 0 yields

$$
g_{0} L=\ln \left(\frac{\beta_{2}^{+}}{\beta_{1}^{+}}\right)+\beta_{2}^{+}-\beta_{1}^{+}-\beta_{0}^{2}\left(\frac{1}{\beta_{2}^{+}}-\frac{1}{\beta_{1}^{+}}\right) .
$$

For the FP laser, this can be solved for the intensity emitted from each facet

$$
\begin{aligned}
& \beta_{1}^{\text {out }}=\left(1-R_{1}\right) \beta_{1}^{-}=\frac{\left(1-R_{1}\right) L\left(g_{0}-g_{0, t h}\right)}{1-R_{1}+\sqrt{R_{1} / R_{2}}-\sqrt{R_{1} R_{2}}}, \\
& \beta_{2}^{\text {out }}=\left(1-R_{2}\right) \beta_{2}^{+}=\frac{\left(1-R_{2}\right) L\left(g_{0}-g_{0, t h}\right)}{1-R_{2}+\sqrt{R_{2} / R_{1}}-\sqrt{R_{1} R_{2}}},
\end{aligned}
$$

and the total emitted intensity

$$
\beta^{\text {out }}=\beta_{1}^{\text {out }}+\beta_{2}^{\text {out }}=L\left(g_{0}-g_{0, t h}\right) .
$$


For the ring laser, it can be shown that the total emitted intensity is also given by Eq. S5 by setting $\beta_{0}=0$ in Eq. S2. Interestingly, the DLA predicts exactly the same total output power (seen by setting $\alpha_{0}=0$ in Eq. 4) as the nonuniform gain saturation treatment in this limit, although the the two theories do not agree on the fraction of the total power coupled out of each mirror of the FP laser. This discrepancy is, however, small in most cases. In particular, there is no discrepancy between the theories for the case of $R_{1}=R_{2}$ (half of the power leaves from each mirror) or when one of the mirrors has unity reflectivity (all of the power is coupled out of the other mirror).

We emphasize again that there is no obvious reason for the DLA to yield the same total output power as the nonuniform treatment when $\alpha_{0}=0$. If nonradiative recombination is neglected [1], then the result can be explained by conservation of energy because the only pathway for electrons to descend from the excited to ground state is through stimulated emission. In other words, the number of photons emitted by the cavity depends only on the total number of electrons passing through the cavity, regardless of the intracavity intensity distribution [1]. Our result is more surprising because it allows electrons to recombine nonradiatively, so this argument does not apply. (Note: to fully explain these statements would require a derivation of the Rigrod equation from rate equations, which is not difficult but outside the scope of our paper. For the interested reader, we also point out that we use a linear relationship between $g_{0}$ and carrier density whereas [1] uses a logarithmic relationship.)

\section{WHEN IS THE ASYMPTOTIC REGIME REACHED IN THE RING LASER?}

In Sec. 3A, we derived the output intensity of the ring laser in Eq. 12. We took the limit of this equation for $g_{0} \gg g_{0, t h}$ resulting in Eq. 14, and called this the asymptotic regime because the output intensity approaches an asymptote. However, it turns out that $g_{0}$ does not even need to be too much greater than $g_{0, t h}$ before the asymptotic regime is reached. To quantify how large $g_{0}$ must be, we look at the ratio of the output power given by the exact expression of Eq. 12 to that of the asymptotic expression of Eq. $14, \beta^{\text {out }} /\left(\left.\beta^{\text {out }}\right|_{g_{0} \gg g_{0, t h}}\right)$, and define $g_{0}^{\text {asmp }}$ as the gain above which this ratio is greater than 0.95 . (Our choice of 0.95 is arbitrary, but is a good indicator for when the exact solution approaches the asymptotic solution.) In Figs. $\mathrm{S} 1$ (a) and S1(b), $g_{0}^{\text {asmp }} / g_{0, t h}$ is plotted against $R$ for several different values of $\alpha_{0} L$. The most striking conclusion is that provided $R>0.01$ (a condition satisfied by nearly all practical lasers), the asymptotic theory becomes very accurate for $g_{0}>2.6 g_{0, t h}$. In Fig. S1(a), we see that as $\alpha_{0} L$ increases from 0.1 to 2 at fixed $R, g_{0}^{\text {asmp }} / g_{0, t h}$ increases as well. However, for $\alpha_{0} L \geq 3$ as shown in Fig. S1(b), this behavior changes, and $g_{0}^{\text {asmp }} / g_{0, t h}$ instead decreases monotonically with increasing $\alpha_{0} L$ at fixed $R$. Combining the information in Figs. 3 and S1(b), we reach an impor-
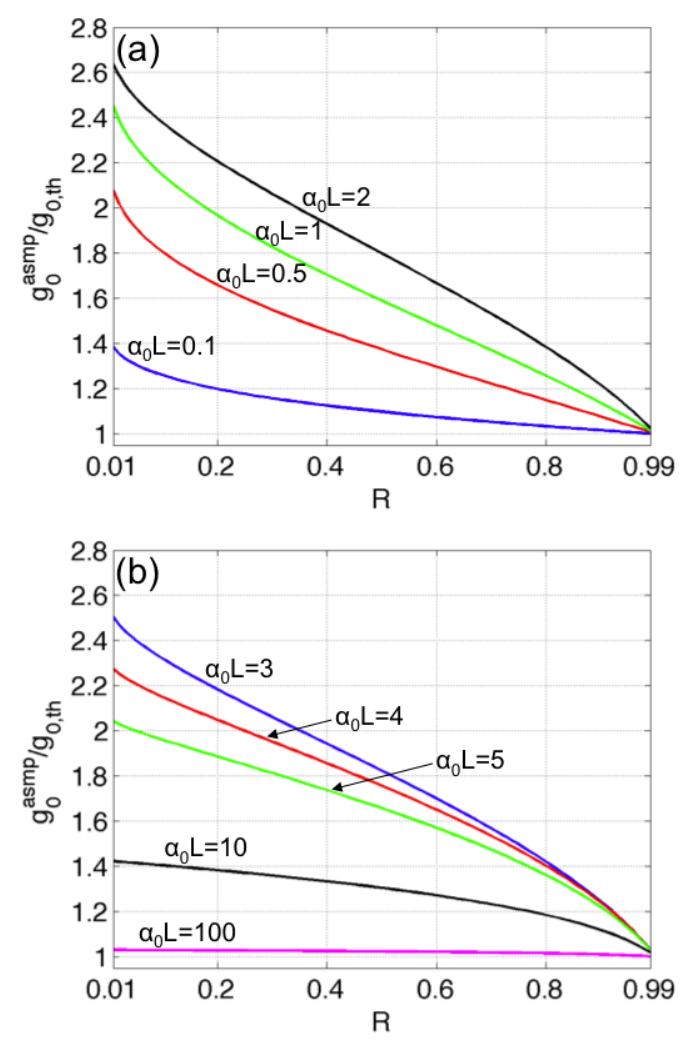

Fig. S1. The value $g_{0}^{\text {asmp }} / g_{0, t h}$-the gain at which the slope efficiency reaches $95 \%$ of its asymptotic valueis plotted for values of (a) $\alpha_{0} L \leq 2$ and (b) $\alpha_{0} L \geq 3$, which shows that $g_{0}^{\text {asmp }}$ decreases monotonically with increasing $\alpha_{0} L$ beyond $\alpha_{0} L=3$.

tant conclusion. As $\alpha_{0} L$ increases beyond $\approx 3$, two things happen: 1) the asymptotic slope efficiency becomes significantly smaller than the threshold slope efficiency, and 2) the asymptotic regime is reached even closer to threshold.

\section{VARIATION OF INTRACAVITY INTENSITY}

The purpose of this supplement is to better understand the variation of the total intensity $\beta^{+}+\beta^{-}$within the laser cavity. For the ring laser this is quite simple since $\beta^{-}=0$, so the maximum and minimum occur at the facets, and their ratio is given by

$$
\left.\frac{\left(\beta^{+}+\beta^{-}\right)_{\min }}{\left(\beta^{+}+\beta^{-}\right)_{\max }}\right|_{\text {ring }}=R .
$$

For the FP laser, recall that $\beta^{+} \beta^{-}=\beta_{0}^{2}$, so the total intensity can be written as

$$
\beta^{+}+\beta^{-}=\beta^{+}+\beta_{0}^{2} / \beta^{+} .
$$

The minimum total intensity is found by differentiating Eq. S7 and occurs at the position where the two counterpropagating waves have equal intensity, i.e., $\beta^{+}=\beta^{-}=$ $\beta_{0}$, and therefore $\left(\beta^{+}+\beta^{-}\right)_{\min }=2 \beta_{0}$. The maximum 
intensity can be found from Eq. S7 to occur at the facet of lower reflectivity, which we take to be $R_{2}$, and is given by $\left(\beta^{+}+\beta^{-}\right)_{\max }=\beta_{0}\left(1+R_{2}\right) / \sqrt{R_{2}}$. Thus, the total intensity variation in the FP laser is given by

$$
\left.\frac{\left(\beta^{+}+\beta^{-}\right)_{\min }}{\left(\beta^{+}+\beta^{-}\right)_{\max }}\right|_{\mathrm{FP}}=\frac{2 \sqrt{R_{2}}}{1+R_{2}},
$$

where $R_{2}$ is the facet of lower reflectivity. It is interesting to note that this variation in total intensity is independent of $g_{0}$ or $\alpha_{0}$-it is determined solely by the facet reflectivities. A ratio closer to 1 indicates a more uniform intensity in the cavity. Equation S8 is plotted in [2] (but was derived here under more general considerations). By comparing Eqs. S6 and S8, one finds that the intensity in the FP is always more uniform than in the ring, and in most cases substantially so. Physically, the reason for this is of course that the intensity in the FP laser is shared between two counter-propagating waves which grow in opposite directions and strongly cross-saturate each other.

For fixed $R=\sqrt{R_{1} R_{2}}$, the most uniform distribution is achieved when $R_{1}=R_{2}$. As $R_{1}$ increases and $R_{2}$ decreases, the intensity becomes more nonuniform. The least uniform distribution occurs for $R_{1}=1$ and the minimum value of $R_{2}=R^{2}$. This is the closest that the FP laser intensity distribution can come to resembling the ring laser intensity distribution-for a given $R$-because the right-propagating intensity $\beta^{+}$is as large as possible relative to the left-propagating $\beta^{-}$. In this case, the ratio of Eq. $S 6$ to Eq. $S 8$ is $\left(1+R_{2}\right) / 2$, which tells us that for small $R_{2}$ the intensity within the FP is still about twice as uniform as that of the ring.

\section{REFERENCES}

1. B. Ryvkin and E. Avrutin, "Spatial hole burning in high-power edge-emitting lasers: A simple analytical model and the effect on laser performance," J. Appl. Phys. 109, 043101 (2011).

2. W. Rigrod, "Saturation Effects in High-Gain Lasers," J. Appl. Phys. 36, 2487-2490 (1965). 